\title{
Adleman-Manders-Miller Root Extraction Method Revisited
}

\author{
Zhengjun Cao*, Qian Sha, Xiao Fan \\ Department of Mathematics, Shanghai University, Shanghai, China. \\ * caozhj@shu.edu.cn
}

\begin{abstract}
In 1977, Adleman, Manders and Miller had briefly described how to extend their square root extraction method to the general $r$ th root extraction over finite fields, but not shown enough details. Actually, there is a dramatic difference between the square root extraction and the general $r$ th root extraction because one has to solve discrete logarithms for $r$ th root extraction. In this paper, we clarify their method and analyze its complexity. Our heuristic presentation is helpful to grasp the method entirely and deeply.
\end{abstract}

Keywords. square root extraction, $r$ th root extraction

\section{Introduction}

Root extraction is a classical problem in computers algebra. It is essential to cryptosystems based on elliptic curves [2]. There are several efficient probabilistic algorithms for square root extraction in finite fields, such as Cipolla-Lehmer [6, 7, Tonelli-Shanks [10, 12] and AdlemanManders-Miller 11. All of them require a quadratic nonresidue as an additional input. In 2004, Müller investigated this topic in Ref. [8]. In 2011, Sze [11] presented a novel idea to compute square roots over finite fields, without being given any quadratic nonresidue, and without assuming any unproven hypothesis.

Adleman-Manders-Miller square root extraction method can be extended to solve the general $r$ th root extraction problem. In recent, Nishihara et al. [9] have specified the Adleman-MandersMiller method for cube root extraction. Barreto and Voloch [2] proposed an efficient algorithm to compute $r$ th roots in $F_{p^{m}}$ for certain choices of $m$ and $p$. Besides, it requires that $r \| p-1$ and $(m, r)=1$, where the notation $a^{b} \| c$ means that $a^{b}$ is the highest power of $a$ dividing $c$.

The basic idea of Adleman-Manders-Miller square root extraction in $F_{p}$ can be described as follows. Write $p-1$ in the form $2^{t} \cdot s$, where $s$ is odd. Given a quadratic residue $\delta$ and a quadratic nonresidue $\rho$, we have

$$
\left(\delta^{s}\right)^{2^{t-1}} \equiv 1(\bmod p), \quad\left(\rho^{s}\right)^{2^{t-1}} \equiv-1(\bmod p)
$$


If $t \geq 2$, then $\left(\delta^{s}\right)^{2^{t-2}}(\bmod p) \in\{1,-1\}$. Take $k_{1}=0$ or 1 such that

$$
\left(\delta^{s}\right)^{2^{t-2}}\left(\rho^{s}\right)^{2^{t-1} \cdot k_{1}} \equiv 1(\bmod p)
$$

Since $\left(\delta^{s}\right)^{2^{t-3}}\left(\rho^{s}\right)^{2^{t-2} \cdot k_{1}}(\bmod p) \in\{1,-1\}$, take $k_{2}=0$ or 1 such that

$$
\left(\delta^{s}\right)^{2^{t-3}}\left(\rho^{s}\right)^{2^{t-2} \cdot k_{1}}\left(\rho^{s}\right)^{2^{t-1} \cdot k_{2}} \equiv 1(\bmod p)
$$

Likewise, we can obtain $k_{3}, \cdots, k_{t-1} \in\{0,1\}$ such that

$$
\left(\delta^{s}\right)\left(\rho^{s}\right)^{2 \cdot k_{1}+2^{2} \cdot k_{2}+\cdots+2^{t-1} \cdot k_{t-1}} \equiv 1(\bmod p)
$$

Thus, we have

$$
\left(\delta^{\frac{s+1}{2}}\right)^{2}\left(\left(\rho^{s}\right)^{k_{1}+2 \cdot k_{2}+\cdots+2^{t-2} \cdot k_{t-1}}\right)^{2} \equiv \delta(\bmod p)
$$

It should be stressed, however, that there is a dramatic difference between the square root extraction and the general $r$ th root extraction. Write $p-1$ in the form $r^{t} \cdot s$, where $(r, s)=1$. Given a $r$ th residue $\delta$ and a $r$ th nonresidue $\rho$, we have

$$
\left(\delta^{s}\right)^{r^{t-1}} \equiv 1(\bmod p), \quad\left(\rho^{s}\right)^{r^{t-1}} \not \equiv 1(\bmod p)
$$

Since $\left(\delta^{s}\right)^{r^{t-2}}(\bmod p)$ is a root of the equation $X^{r} \equiv 1(\bmod p)$ and the equation has $r$ different roots (these roots can be represented by $\left(\rho^{s}\right)^{k_{i} \cdot r^{t-1}}, k_{i} \in\{0,1, \cdots, r-1\}$ ), it becomes difficult to find $k_{1}$ such that

$$
\left(\delta^{s}\right)^{r^{t-2}}\left(\rho^{s}\right)^{r^{t-1} \cdot k_{1}} \equiv 1(\bmod p)
$$

In 1977, Adleman, Manders and Miller [1] had presented a brief description on how to extend their square root extraction method to the general $r$ th root extraction over finite fields, but not shown enough details. By the way, it is the only known method for the general $r$ th root extraction over finite fields. In this paper, we clarify their method and analyze its complexity.

\section{Preliminary}

Let $Z_{n}=\{0,1, \cdots, n-1\}$ be the set of all numbers smaller than $n, Z_{n}^{*}=\{x \mid 1 \leq x \leq$ $n$ and $\operatorname{gcd}(x, n)=1\}$ be the set of numbers in $Z_{n}$ that are coprime to $n$. The following definitions and results can be found in Ref. 4 .

Definition 1. A residue $a \in Z_{n}^{*}$ is said to be a quadratic residue if there exists some $x \in Z_{n}^{*}$ such that $x^{2} \equiv a(\bmod n)$. If a is not a quadratic residue, then it is referred to as a quadratic non-residue.

Theorem 2. (Euler's Criterion) For prime $p$, an element $a \in Z_{p}^{*}$ is a quadratic residue if and only if $a^{\frac{p-1}{2}} \equiv 1(\bmod p)$. 
Definition 3. (Legendre Symbol) For any prime $p$ and $a \in Z_{p}^{*}$, we define the Legendre symbol

$$
\left[\frac{a}{p}\right]= \begin{cases}1 & \text { if } a \text { is a quadratic residue }(\bmod p) \\ -1 & \text { if } a \text { is a quadratic non-residue }(\bmod p)\end{cases}
$$

For an integer $a$, we define $\log (a)$ to be the number of bits in the binary representation of $|a| ;$ more precisely,

$$
\log (a)= \begin{cases}\left\lfloor\log _{2}|a|\right\rfloor+1 & \text { if } a \neq 0 \\ 1 & \text { if } a=0\end{cases}
$$

Given $a \in Z_{n}$ and a non-negative integer $e$, the repeated-squaring algorithm computes $a^{e}(\bmod n)$ using just $\mathcal{O}(\log (e))$ multiplications in $Z_{n}$, thus taking time $\mathcal{O}\left(\log (e) \log ^{2} n\right)$. Therefore, we have the following result:

Proposition 4. For an odd prime $p$, we can test whether an integer a is a quadratic residue modulo $p$ by either performing the exponentiation $a^{\frac{(p-1)}{2}}(\bmod p)$ or by computing the Legendre symbol $\left[\frac{a}{p}\right]$. Assume that $0<a<p$. Using a standard repeated squaring algorithm, the former method takes time $\mathcal{O}\left(\log ^{3} p\right)$, while using Euclidean-like algorithm, the latter method takes time $\mathcal{O}\left(\log ^{2} p\right)$.

Proof. See [5].

Let $R$ be a ring. Let us define the length of a polynomial $f(X) \in R[X]$, denoted by $\log (f)$, to be the length of its coefficient vector; more precisely, we define

$$
\log (f)= \begin{cases}\operatorname{deg}(f)+1 & \text { if } f \neq 0 \\ 1 & \text { if } f=0\end{cases}
$$

Analogous to algorithms for modular integer arithmetic, we can also do arithmetic in the residue class ring $R[X] /(f)$, where $f \in R[X]$ is a polynomial of $\operatorname{deg}(f)>0$ whose leading coefficient $\operatorname{lc}(f)$ is a unit.

Proposition 5. Let $R[X] /(f)$ be a residue class ring, where $f \in R[X]$ is a polynomial of $\operatorname{deg}(f)>0$ whose leading coefficient $\operatorname{lc}(f)$ is a unit. Given $g \in R[X] /(f)$ and a non-negative exponent $e$, using repeated-squaring algorithm we can compute $g^{e}$ taking $\mathcal{O}\left(\log (e) \operatorname{deg}(f)^{2}\right)$ operations in $R$.

Proof. See [3].

Notice that using a standard representation for $F_{p}$, each operation in $F_{p}$ takes time $\mathcal{O}\left(\log ^{2} p\right)$.

\section{Adleman-Manders-Miller square root extraction method}

The Adleman-Manders-Miller square root extraction method requires a quadratic non-residue as an additional input. We classify the method into two kinds because there is a gap between 
the base field $F_{p}$ and the extension $F_{p^{m}}$ to test whether an element is a quadratic non-residue.

\subsection{Adleman-Manders-Miller square root extraction method in $F_{p}$}

Consider the problem to find a solution to the congruence $X^{2} \equiv \delta(\bmod p)$ over finite field $F_{p}$, where $p$ is an odd prime.

Adleman, Manders and Miller [1] proposed an algorithm to solve the problem. Their square root extraction method is based on the following facts. Write $p-1$ in the form $2^{t} \cdot s$, where $s$ is odd. Given a quadratic residue $\delta$ and a quadratic nonresidue $\rho$, we have

$$
\left(\delta^{s}\right)^{2^{t-1}} \equiv 1(\bmod p), \quad\left(\rho^{s}\right)^{2^{t-1}} \equiv-1(\bmod p)
$$

If $t=1$, then $\delta^{s} \equiv 1(\bmod p)$. Hence, we have $\left(\delta^{\frac{s+1}{2}}\right)^{2} \equiv \delta(\bmod p)$. It means that $\delta^{\frac{s+1}{2}}$ is a square root of $\delta$. In this case, it only takes time $\mathcal{O}\left(\log (s) \log ^{2} p\right)$.

If $t \geq 2$, then $\left(\delta^{s}\right)^{2^{t-2}}(\bmod p) \in\{1,-1\}$. Take $k_{1}=0$ or 1 such that

$$
\left(\delta^{s}\right)^{2^{t-2}}\left(\rho^{s}\right)^{2^{t-1} \cdot k_{1}} \equiv 1(\bmod p)
$$

Take $k_{2}=0$ or 1 such that

$$
\left(\delta^{s}\right)^{2^{t-3}}\left(\rho^{s}\right)^{2^{t-2} \cdot k_{1}}\left(\rho^{s}\right)^{2^{t-1} \cdot k_{2}} \equiv 1(\bmod p)
$$

Likewise, we obtain $k_{3}, \cdots, k_{t-1} \in\{0,1\}$ such that

$$
\left(\delta^{s}\right)\left(\rho^{s}\right)^{2 \cdot k_{1}+2^{2} \cdot k_{2}+\cdots+2^{t-1} \cdot k_{t-1}} \equiv 1(\bmod p)
$$

Finally, we have

$$
\left(\delta^{\frac{s+1}{2}}\right)^{2}\left(\left(\rho^{s}\right)^{k_{1}+2 \cdot k_{2}+\cdots+2^{t-2} \cdot k_{t-1}}\right)^{2} \equiv \delta(\bmod p)
$$

To find a quadratic non-residue $\rho$, it requires to check that $\left[\frac{\rho}{p}\right] \neq 1$. The computation takes time $\mathcal{O}\left(\log ^{2} p\right)$. If we do this for more than $\mathcal{O}(1) \log p$ different randomly chosen $\rho$, then with probability $>1-\left(\frac{1}{p}\right)^{\mathcal{O}(1)}$ at least one of them will give a quadratic non-residue. Thus, to find a quadratic nonresidue $\rho$, it takes expected time $\mathcal{O}\left(\log ^{3} p\right)$. To compute $b^{2^{t-i-1}}(\bmod p)$, it takes time $\mathcal{O}\left((t-i-1) \log ^{2} p\right)$. Since there are $1+2+\cdots+(t-1)=\frac{t(t-1)}{2}$ steps, the loop takes time $\mathcal{O}\left(t^{2} \log ^{2} p\right)$. Thus, the total estimate is $\mathcal{O}\left(\log ^{3} p+t^{2} \log ^{2} p\right)$. At worst (if almost all of $p-1$ is a power of 2$)$, this is $\mathcal{O}\left(\log ^{4} p\right)$.

\subsection{Adleman-Manders-Miller square root extraction method in $F_{p^{m}}$}

As we mentioned before, the Adleman-Manders-Miller method in the extension field $F_{p^{m}}$ differs from the method in the base field $F_{p}$ because one can not determine a quadratic non-residue by computing the Legendre Symbol. 
Table 1: Adleman-Manders-Miller square root extraction algorithm in $F_{p}$

Input: Odd prime $p$ and a quadratic residue $\delta$.

Output: A square root of $\delta$.

Step 1: Choose $\rho$ uniformly at random from $F_{p}^{*}$.

Compute $\left[\frac{\rho}{p}\right]$ using Euclidean-like algorithm.

Step 2: if $\left[\frac{\rho}{p}\right]=1$, go to Step 1 .

Step 3: Compute $t, s$ such that $p-1=2^{t} s$, where $s$ is odd.

Compute $a \leftarrow \rho^{s}, b \leftarrow \delta^{s}, h \leftarrow 1$.

Step 4: for $i=1$ to $t-1$

$$
\begin{aligned}
& \text { compute } d=b^{2^{t-1-i}} \\
& \text { if } d=1, k \leftarrow 0 \\
& \text { else } k \leftarrow 1 \\
& b \leftarrow b \cdot\left(a^{2}\right)^{k}, h \leftarrow h \cdot a^{k} \\
& a \leftarrow a^{2}
\end{aligned}
$$

end for

Step 5: return $\delta^{\frac{s+1}{2}} \cdot h$

Set $q=p^{m}$. To find a quadratic non-residue $\rho$, it requires to check that $\rho^{\frac{q-1}{2}} \neq 1$. The computation takes time $\mathcal{O}\left(\log ^{3} q\right)$. If we do this for more than $\mathcal{O}(1) \log q$ different randomly chosen $\rho$, then with probability $>1-\left(\frac{1}{q}\right)^{\mathcal{O}(1)}$ at least one of them will give a quadratic nonresidue. Thus, to find a quadratic nonresidue $\rho$, it takes expected time $\mathcal{O}\left(\log ^{4} q\right)$.

To compute $b^{2^{t-i-1}}$, it takes time $\mathcal{O}\left((t-i-1) \log ^{2} q\right)$. Since there are $1+2+\cdots+(t-1)$ steps, the loop takes time $\mathcal{O}\left(t^{2} \log ^{2} q\right)$. Thus, the final estimate is $\mathcal{O}\left(\log ^{4} q+t^{2} \log ^{2} q\right)$.

\section{Adleman-Manders-Miller cubic root extraction method}

In 2009, Nishihara et al. 9] specified the Adleman-Manders-Miller method for cube root extraction. See the following description.

Set $q=p^{m}$. The cubic root extraction algorithm takes time $\mathcal{O}\left(\log ^{4} q+t^{2} \log ^{2} q\right)$. As for this claim, we refer to the complexity analysis of Adleman-Manders-Miller square root extraction algorithm in Section 3.2. 
Table 2: Adleman-Manders-Miller square root extraction algorithm in $F_{p^{m}}$

Input: Odd prime $p$, a positive integer $m$ and a quadratic residue $\delta$.

Output: A square root of $\delta$.

Step 1: Choose $\rho$ uniformly at random from $F_{p^{m}}^{*}$.

Step 2: if $\rho^{\frac{p^{m}-1}{2}}=1$, go to Step 1 .

Step 3: Compute $t, s$ such that $p^{m}-1=2^{t} s$, where $s$ is odd.

Compute $a \leftarrow \rho^{s}, b \leftarrow \delta^{s}, h \leftarrow 1$.

Step 4: for $i=1$ to $t-1$

compute $d=b^{2^{t-1-i}}$

if $d=1, k \leftarrow 0$

else $k \leftarrow 1$

$b \leftarrow b \cdot\left(a^{2}\right)^{k}, h \leftarrow h \cdot a^{k}$

$a \leftarrow a^{2}$

end for

Step 5: return $\delta^{\frac{s+1}{2}} \cdot h$

\section{Specification of Adleman-Manders-Miller $r$ th Root Extrac- tion Method}

Consider the general problem to find a solution to $X^{r}=\delta$ in $F_{q}$. Clearly, it suffices to consider the following two cases:

$$
\text { (1) }(r, q-1)=1 ; \quad(2) r \mid q-1 .
$$

If $(r, q-1)=1$, then $\delta^{r^{-1}}$ is a $r$ th root of $\delta$. Therefore, it suffices to consider the case that $r \mid q-1$.

Adleman, Manders and Miller [1] had mentioned how to extend their square root extraction method to $r$ th root extraction, but not specified it. We now clarify it as follows.

If $r \mid q-1$, we write $p-1$ in the form $r^{t} \cdot s$, where $(s, r)=1$. Given a $r$ th residue $\delta$, we have $\left(\delta^{s}\right)^{r^{t-1}}=1$. Since $(s, r)=1$, it is easy to find the least nonnegative integer $\alpha$ such that $s \mid r \alpha-1$. Hence,

$$
\left(\delta^{r \alpha-1}\right)^{r^{t-1}}=1
$$

If $t-1=0$, then $\delta^{\alpha}$ is a $r$ th root of $\delta$. From now on, we assume that $t \geq 2$.

Given a $r$ th non-residue $\rho \in F_{q}$, we have

$$
\left(\rho^{s}\right)^{i \cdot r^{t-1}} \neq\left(\rho^{s}\right)^{j \cdot r^{t-1}} \text { where } i \neq j, i, j \in\{0,1, \cdots, r-1\}
$$


Table 3: Adleman-Manders-Miller cubic root extraction algorithm in $F_{p^{m}}$

Input: Odd prime $p$, a positive integer $m$ and a cubic residue $\delta$.

Output: A cubi root of $\delta$.

Step 1: Choose $\rho$ uniformly at random from $F_{p^{m}}^{*}$.

Step 2: if $\rho^{\frac{p^{m}-1}{3}}=1$, go to Step 1 .

Step 3: Compute $t, s$ such that $p^{m}-1=3^{t} s$, where $s=3 l \pm 1$.

Compute $a \leftarrow \rho^{s}, a^{\prime} \leftarrow \rho^{3^{t-1} \cdot s}, b \leftarrow \delta^{s}, h \leftarrow 1$.

Step 4: for $i=1$ to $t-1$

compute $d=b^{3^{t-1-i}}$

if $d=1, k \leftarrow 0$,

else if $d=a^{\prime}, k \leftarrow 2$

else $k \leftarrow 1$

$b \leftarrow b \cdot\left(a^{3}\right)^{k}, h \leftarrow h \cdot a^{k}$

$a \leftarrow a^{3}$

end for

Step 5: $r \leftarrow \delta^{l} h$

if $s=3 l+1, r \leftarrow r^{-1}$

return $r$

Set

$$
K_{i}=\left(\rho^{s}\right)^{i \cdot r^{t-1}} \text { and } K=\left\{K_{0}, K_{1}, \cdots, K_{r-1}\right\}
$$

It is easy to find that all $K_{i}$ satisfy $X^{r}=1$. Since

$$
\left(\left(\delta^{r \alpha-1}\right)^{r^{t-2}}\right)^{r}=1
$$

there is a unique $j_{1} \in\{0,1, \cdots, r-1\}$ such that

$$
\left(\delta^{r \alpha-1}\right)^{r^{t-2}}=K_{r-j_{1}}
$$

where $K_{r}=K_{0}$. Hence,

$$
\left(\delta^{r \alpha-1}\right)^{r^{t-2}} K_{j_{1}}=1
$$

That is

$$
\left(\delta^{r \alpha-1}\right)^{r^{t-2}}\left(\rho^{s}\right)^{j_{1} \cdot r^{t-1}}=1
$$

By the way, to obtain $j_{1}$ one has to solve a discrete logarithm. 
Likewise, there is a unique $j_{2} \in\{0,1, \cdots, r-1\}$ such that

$$
\left(\delta^{r \alpha-1}\right)^{r^{t-3}}\left(\rho^{s}\right)^{j_{1} \cdot r^{t-2}}\left(\rho^{s}\right)^{j_{2} \cdot r^{t-1}}=1
$$

Consequently, we can obtain $j_{1}, \cdots, j_{t-1}$ such that

$$
\left(\delta^{r \alpha-1}\right)\left(\rho^{s}\right)^{j_{1} \cdot r}\left(\rho^{s}\right)^{j_{2} \cdot r^{2}} \cdots\left(\rho^{s}\right)^{j_{t-1} \cdot r^{t-1}}=1
$$

Thus, we have

$$
\left(\delta^{\alpha}\right)^{r}\left(\left(\rho^{s}\right)^{j_{1}+j_{2} \cdot r+\cdots j_{t-1} \cdot r^{t-2}}\right)^{r}=\delta
$$

It means that

$$
\delta^{\alpha}\left(\rho^{s}\right)^{j_{1}+j_{2} \cdot r+\cdots j_{t-1} \cdot r^{t-2}}
$$

is a $r$ th root of $\delta$.

Table 4: Adleman-Manders-Miller $r$ th root extraction algorithm in $F_{q}$

Input: $F_{q}$ and a $r$ th residue $\delta, r \mid q-1$.

Output: A $r$ th root of $\delta$.

Step 1: Choose $\rho$ uniformly at random from $F_{q}^{*}$.

Step 2: if $\rho^{\frac{q-1}{r}}=1$, go to Step 1 .

Step 3: Compute $t, s$ such that $q-1=r^{t} s$, where $(r, s)=1$.

Compute the least nonnegative integer $\alpha$ such that $s \mid r \alpha-1$.

Compute $a \leftarrow \rho^{r-1} s, b \leftarrow \delta^{r \alpha-1}, c \leftarrow \rho^{s}, h \leftarrow 1$

Step 4: for $i=1$ to $t-1$

compute $d=b^{r^{t-1-i}}$

if $d=1, j \leftarrow 0$,

else $j \leftarrow-\log _{a} d$ (compute the discrete logarithm)

$b \leftarrow b\left(c^{r}\right)^{j}, h \leftarrow h c^{j}$

$c \leftarrow c^{r}$

end for

Step 5: return $\delta^{\alpha} \cdot h$

\section{Complexity analysis of Adleman-Manders-Miller $r$ th Root Ex- traction Method}

We now discuss the time estimate for this $r$ th root extraction algorithm. 
To find a $r$ th non-residue $\rho$, it requires to check that $\rho^{\frac{q-1}{r}} \neq 1$. The computation takes time $\mathcal{O}\left(\log ^{3} q\right)$. If we do this for more than $\mathcal{O}(1) \log q$ different randomly chosen $\rho$, then with probability $>1-\left(\frac{1}{q}\right)^{\mathcal{O}(1)}$ at least one of them will give a $r$ th non-residue. Therefore, the expected time of finding a $r$ th non-residue is $\mathcal{O}\left(\log ^{4} q\right)$.

The work done outside the loop amounts to just a handful of exponentiations. Hence, it takes time $\mathcal{O}\left(\log ^{3} q\right)$. To compute $b^{r^{t-i-1}}$, it takes time $\mathcal{O}\left((t-i-1) \log r \log ^{2} q\right)$. Since there are $1+2+\cdots+(t-1)$ steps, it takes time $\mathcal{O}\left(t^{2} \log r \log ^{2} q\right)$.

To compute the discrete $\operatorname{logarithm} \log _{a} d$, it takes time $\mathcal{O}\left(r \log ^{2} q\right)$ using brute-force search. Since there are $t-1$ discrete logarithms at worst, it takes time $\mathcal{O}\left(\operatorname{tr} \log ^{2} q\right)$.

Thus, the final estimate is $\mathcal{O}\left(\log ^{4} q+r \log ^{3} q\right)$. Notice that the algorithm can not run in polynomial time if $r$ is sufficiently large.

\section{Conclusion}

The basic idea of Adleman-Manders-Miller root extraction method and its complexity analysis have not specified in the past decades. In this paper, we clarify the method and analyze its complexity. We think our heuristic presentation is helpful to grasp the method entirely and deeply.

Acknowledgements. We thank the anonymous referees' for their detailed suggestions. This work is supported by the National Natural Science Foundation of China (Project 60873227, 11171205), and the Key Disciplines of Shanghai Municipality (S30104).

\section{References}

[1] Adleman L., Manders K., Miller G.: On Taking Roots in Finite Fields. In: Proceedings of the 18th IEEE Symposium on Foundations of Computer Science, pp. 175-177. IEEE Press, New York (1977)

[2] Barreto P., Voloch J.: Efficient Computation of Roots in Finite Fields. Designs, Codes and Cryptography, 39, 275-280 (2006)

[3] Gathen J., Gerhard J.: Modern Computer Algebra, 2nd ed., Cambridge University Press (2003)

[4] Lidl R., Niederreiter H.: Introduction to finite fields and their applications, Cambridge University Press (1986)

[5] Shoup V.: A Computational Introduction to Number Theory and Algebra. Cambridge University Press (2005) 
[6] Cipolla M.: Un metodo per la risolutione della congruenza di secondo grado, Rendiconto dell'Accademia Scienze Fisiche e Matematiche, Napoli, Ser. 3, Vol. IX, pp. 154-163 (1903)

[7] Lehmer D.: Computer technology applied to the theory of numbers, Studies in Number Theory, Englewood Cliffs, NJ: Pretice-Hall, pp. 117-151 (1969)

[8] Müller S.: On the computation of square roots in finite fields, Designs, Codes and Cryptography, 31, 301-312 (2004)

[9] Nishihara N., Harasawa R., Sueyoshi Y., and Kudo A.: A remark on the computation of cube roots in finite fields, eprint.iacr.org/2009/457

[10] Shanks D.: Five Number-theoretic Algorithms. In: Proc. 2nd Manitoba Conf., pp. 51-70. Numer. Math. (1972)

[11] Sze T., On taking square roots without quadratic nonresidues over finite fields, Mathematics of Computation, 80, 1797-1811 (2011)

[12] Tonelli A.: Bemerkungüber die Auflösung quadratischer Congruenzen. Nachrichten der Akademie der Wissenschaften in Göttingen. 344-346 (1891) 